\title{
Alimentación total oral en prematuros menores de 30 semanas de gestación internados en Unidad de Cuidado Intensivo Neonatal
}

\author{
DOI: $10.46981 / \mathrm{sfjhv2n2-002}$
}

Received in: January 1st, 2020

Accepted in: March 30th, 2020

\author{
Nestor Hugo Rosendo \\ Physical Therapy- Pediatric and Neonatal Physical Therapy Specialist-UBA \\ Institution: University U.B.A- Children's Hospital Sor María Ludovica \\ Address: 50 n 1067 Villa Elisa Bs As, Argentina \\ E-mail: nhrosendo@gmail.com
}

\author{
Nancy Analía Aparicio \\ Medical-Master in Health Economics and Administration of Health Organizations- UNLP \\ Institution: University U.N.L.P- Children's Hospital Sor María Ludovica \\ Address: Calle: 77 bis No 282 La Plata, Bs As, Argentina \\ E-mail: osimamu@hotmail.com
}

\section{RESUMEN}

Alimentarse por vía oral independiente sin necesidad del gavage es un gran desafío para todos los bebés prematuros extremos y un criterio muy importante para considerar al alta hospitalaria. En este trabajo estudiamos retrospectivamente una población de bebés prematuros menores de 30 semanas de gestación y registramos a que edad comenzaron a alimentarse por vía oral y a que edad alcanzaron el volumen total como variables principales. Obtuvimos un resultado relativamente alto que intentaremos mejorar en próximos trabajos de intervención.

\section{INTRODUCCIÓN}

La alimentación por vía oral es un proceso muy complejo para los bebés prematuros. Durante la misma suelen presentar dificultades debido a que presentan sistemas inmaduros entre los que se destacan el cardiorrespiratorio, el sistema nervioso central y en particular el sistema sensoriomotor oral que está atravesando su período crítico de plasticidad. Esta dificultad generalmente se traduce en una estadía más prolongada en el hospital.(Carvajal Beatriz, 2010)(Greene et al., 2016)

Se considera que un bebé alcanza la alimentación total oral (ATO) cuando es capaz de recibir todo el volumen por vía oral durante 24 horas sin necesidad de utilizar gavage, ya sea tomando el pecho o biberón. (McCain, 2003)Este proceso de transición de la alimentación involucra el desarrollo de variados sistemas como el autónomo, sensorial, motor, estados de alerta e interacción con el entorno y la progresiva madurez de los mismos.Estos sistemas a su vez son enormemente influenciados por el impacto ambiental en la Unidad de Cuidados Intensivo Neonatal (UCIN) y la gran dependencia e interacción que pueda recibir de su madre y demás cuidadores como son los miembros del equipo de salud que trabajan en torno a él durante su internación en la Unidad de Cuidados Intensivos Neonatales. (Als et al., 2004) (Browne \& Ross, 2011) 


\section{MARCO TEÓRICO}

Varios autores han estudiado la maduración de la succión, respiración y deglución en bebés prematuros y asumen que la coordinación entre respiración y deglución no se encuentra completamente organizada hasta la semana 34 de Edad post Concepcional (EPC) (Mizuno \& Ueda, 2003)

Otros estudian cómo realizar la transferencia de la alimentación por sonda nasogástrica a la alimentación oral en bebés prematuros con distintos enfoques y diferentes resultados (Medeiros et al., 2011),(Lau \& Smith, 2011)(Scochi et al., 2010)

Es de suma importancia valorar el estado de salud del bebé y ofrecer experiencias orales positivas (Thoyre et al., 2005)(Browne \& Ross, 2011) El recién nacido prematuro (RNPT) es un bebé altamente dependiente de los cuidados que recibe por su propia inmadurez y asistirlo en el proceso de transición desde la alimentación enteral por gavage hasta la alimentación oral completa es un gran desafío para la familia y por el equipo de salud de la UCIN ya que es a menudo la competencia final que un RNPT necesita alcanzar antes de ser dado de alta.(Younesian et al., 2015)

Por lo tanto, conocer cuál es la edad postconcepcional en la que los bebés prematuros alcanzan su alimentación oral total por sus propios medios podría ser de gran utilidad para saber cómo implementar algún paquete de medidas tendientes a reducir esos tiempos.(Liu et al., 2013)

La UCIN donde trabajamos es de 3 nivel y cuenta con capacidad para 44 bebés; pertenece a un Hospital Interzonal de Agudos Especializado en Pediatría, que no posee Maternidad por lo tanto todos los niños ingresan por derivación desde otros hospitales de menor complejidad.

\section{OBJETIVO}

Como principal objetivo se realizó un estudio descriptivo observacional retrospectivo para conocer la media de la Edad Post Concepcional (EPC) en que los prematuros internados durante los años 2015 y 2016 nacidos con menos de 30 semanas de edad gestacional (EG), habían comenzado a succionar, respirar y deglutir por vía oral en forma coordinada sin alterar su estabilidad hemodinámica y cuando habían alcanzado la ATO.

Como objetivo secundario se estudió el peso que tenía cada bebe cuando realizó la 1er toma y cuando alcanzó la ATO.

\section{MATERIALES Y MÉTODOS}

Se realizó un análisis retrospectivo de las Historias Clínicas de los recién nacidos menores de 30 semanas de gestación, atendidos entre 2015 y 2016. Se registraron antecedentes perinatales, variables antropométricas y el progreso hacia la alimentación total oral independiente. Las variables continuas se 
presentaron como media \pm desvío estándar (DE) o mediana y rango intercuartílico (RIQ) según correspondiera.

Las variables categóricas se presentaron como porcentajes. Mediante la prueba de Student, se compararon medias de EPC a la primera toma de alimentos y al momento de alimentación total oral con respecto a EG al nacimiento (corte en 27 semanas, para peso al nacer (1000 g), evolución simple o compleja y leucomalacia periventricular (LPV).

\section{RESULTADOS}

Se encontraron 25 H.C. de niños menores de 30 semanas en el período estudiado. La mediana (RIQ) de EG al nacer fue 27 (26-28), y la media \pm DE de peso al nacer, $1031 \pm 46 \mathrm{~g}$. la mediana (RIQ) de días de internación fue 96 (76-111), mientras que la mediana (RIQ) de días con alimentación parenteral fue 14 (11-21). $64 \%$ tuvieron una evolución simple, mientras que 32\% de los niños fueron deprimidos neonatales, $13 \%$ sufrieron hemorragia intracraneana, 16\% LPV y 8\% enterocolitis necrotizante.

Las medias \pm DE de EPC de la primera alimentación fue 39,0 \pm 0,5 y la de alimentación total oral fue 40,5 $\pm 0,6$. Se encontró una media de EPC a la primera toma mayor en niños con LPV (41,6 vs 38,4 semanas, $\mathrm{p}=0,017$ ). Para el resto de las variables estudiadas no se hallaron diferencias significativas.

\section{CONCLUSIONES}

En este trabajo vemos reflejado que la alimentación total oral de los bebés prematuros menores a 30_semanas internados en la UCIN tuvo una media de 40,5 \$ 0,6 semanas de EPC y fue aún más alto en los bebés que padecieron leucomalacia.

Es un valor moderadamente alto que deseamos poder mejorar implementando mejores prácticas basadas en la evidencia.

Sería de vital importancia realizar más estudios en los que se implementen paquetes de intervenciones tendientes a mejorar los procesos que forman parte de esta compleja función de coordinación de succión-deglución-respiración verdaderamente eficiente intentando disminuir los tiempos de internación y favoreciendo la rápida revinculación familiar en un clima hogareño. 


\section{BIBLIOGRAPHY}

Als, H., Duffy, F. H., McAnulty, G. B., Rivkin, M. J., Vajapeyam, S., Mulkern, R. V., Warfield, S. K., Huppi, P. S., Butler, S. C., Conneman, N., Fischer, C., \& Eichenwald, E. C. (2004). Early Experience Alters Brain Function and Structure. Pediatrics, 113(4 I), 846-857. https://doi.org/10.1542/peds.113.4.846

Browne, J. V., \& Ross, E. S. (2011). Eating as a Neurodevelopmental Process for High-Risk Newborns. Clinics in Perinatology, 38(4), 731-743. https://doi.org/10.1016/j.clp.2011.08.004

Carvajal Beatriz. (2010). El progreso de la alimentación oral del recién nacido prematuro. Alimentación oral del recién nacido. Revista Salud, 42(3), 1-13.

Greene, Z., O’Donnell, C. P. F., \& Walshe, M. (2016). Oral stimulation for promoting oral feeding in preterm infants. Cochrane Database of Systematic Reviews, 2016(9). https://doi.org/10.1002/14651858.CD009720.pub2

Lau, C., \& Smith, E. O. (2011). A novel approach to assess oral feeding skills of preterm infants. Neonatology, 100(1), 64-70. https://doi.org/10.1159/000321987

Liu, Y. L., Chen, Y. L., Cheng, I., Lin, M. I., Jow, G. M., \& Mu, S. C. (2013). Early oral-motor management on feeding performance in premature neonates. Journal of the Formosan Medical Association, 112(3), 161-164. https://doi.org/10.1016/j.jfma.2012.08.003

McCain, G. C. (2003). An evidence-based guideline for introducing oral feeding to healthy preterm infants. Neonatal Network: NN, 22(5), 45-50. https://doi.org/10.1891/0730-0832.22.5.45

Mizuno, K., \& Ueda, A. (2003). The maturation and coordination of sucking, swallowing, and respiration in preterm infants. Journal of Pediatrics, 142(1), 36-40. https://doi.org/10.1067/mpd.2003.mpd0312

Scochi, C. G. S., Gauy, J. S., Fujinaga, C. I., Fonseca, L. M. M., \& Zamberlan, N. E. (2010). Transição alimentar por via oral em prematuros de um Hospital Amigo da Criança. Acta Paulista de Enfermagem, 23(4), 540-545. https://doi.org/10.1590/s0103-21002010000400015

Thoyre, S. M., Shaker, C. S., \& Pridham, K. F. (2005). The early feeding skills assessment for preterm infants. Neonatal Network: NN, 24(3), 7-16. https://doi.org/10.1891/0730-0832.24.3.7

Younesian, S., Yadegari, F., \& Soleimani, F. (2015). Impact of oral sensory motor stimulation on feeding performance, length of hospital stay, and weight gain of preterm infants in NICU. Iranian Red Crescent Medical Journal, 17(7). https://doi.org/10.5812/ircmj.17(5)2015.13515 


\section{ANEXOS}

Fig 1. Antecedentes perinatales de la muestra
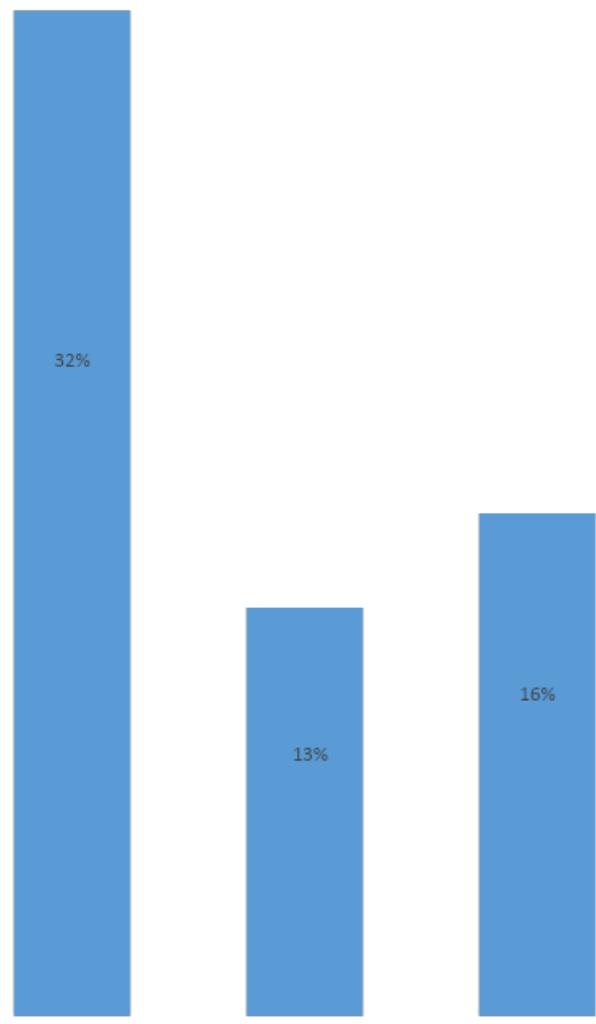

Apgar $<5$

Tabla 1 - Características de la muestra poblacional

\begin{tabular}{|c|c|c|}
\hline \multicolumn{3}{|c|}{ Características de la muestra $\quad(n=25)$} \\
\hline EG & Mediana $=27$ & RIQ 26-28 \\
\hline Peso & Media 1031 & $\mathrm{DE} \pm 46 \mathrm{~g}$ \\
\hline
\end{tabular}

Tabla 2- Resultados

\begin{tabular}{|c|c|c|}
\hline \multicolumn{2}{|c|}{ Logros en alimentación relacionados a EG } \\
\hline EG 1 toma & Media 39 & DE 0,5 \\
\hline EG alimentación total & Media 40,5 & DE 0,6 \\
\hline $\begin{array}{c}\text { EG 1 }{ }^{\text {a }} \text { toma grupo de } \\
\text { condiciones médicas } \\
\text { complejas }\end{array}$ & Media 41,6 & p=0,017 * \\
\hline \multicolumn{2}{|c|}{$*$ Prueba t de Student, vs niños sin LPV. } \\
\hline
\end{tabular}

